\title{
Diagnostic sensitivity of thyroid autoantibodies assessed in a population-based, cross-sectional study in adults
}

\author{
Emanuele Bosi • Roberto Bianchi • Giacomo Ruotolo • \\ Elena Bazzigaluppi • Cristina Belloni • Giliola Calori • \\ Anna Maria Girardi • Maria Paola Garancini • Marina Scavini
}

Received: 10 April 2010 / Accepted: 13 April 2010

(c) Springer-Verlag 2010

\begin{abstract}
The aim of this study was to estimate the diagnostic sensitivity of thyroid autoantibodies in individuals with a case-mix of subjects with thyroid disease representing that of the general population. We measured thyroid microsome (TMA), thyroid peroxidase (TPO), thyroglobulin (TGA) and thyroid-stimulating hormone (TSH) receptor (TRA) autoantibodies in subjects in the bottom (hyperthyroid end) and top (hypothyroid end) four percentiles of the TSH distribution from among participants in a population-based survey of individuals aged $\geq 40$ years (the Cremona Study). TMA and TPO were the most sensitive autoantibodies in subjects in both the bottom percentiles (19.8\% and $18.5 \%$, respectively) and the top percentiles $(51.2 \%$ and $53.8 \%$, respectively) of the TSH distribution. TMA and TPO showed good agreement
\end{abstract}

E. Bosi (凷) - R. Bianchi $\cdot$ M. Scavini

General Medicine, Diabetes \& Endocrinology Unit, San Raffaele Scientific Institute and Vita Salute San Raffaele University, Via Olgettina, 60, 20132 Milan, Italy

Tel.: +39-02-2643-2818

Fax: +39-02-2643-2827

E-mail: bosi.emanuele@hsr.it

E. Bosi · E. Bazzigaluppi · C. Belloni · A.M. Girardi Autoimmunity Unit, Laboraf, San Raffaele Scientific Institute, Milan, Italy

G. Ruotolo · G. Calori

Epidemiology Unit, Cardiovascular Department, San Raffaele

Scientific Institute, Milan, Italy

M.P. Garancini

Medical Direction, San Raffaele Scientific Institute, Milan, Italy (kappa statistics 87.8\%, 95\% CI 80.1-95.5\%) at both ends of the TSH distribution. TGA were the next most sensitive marker, although seldom detected if TMA or TPO were not present. TRA were detected only at the extremes of the TSH distribution (1st percentile, 31.8\%; 100th percentile, $25.0 \%$ ). We conclude that, among a case-mix of individuals with thyroid disease representing that of the general population, TMA and TPO are the most sensitive markers of thyroid disease. TGA only marginally increased the diagnostic sensitivity of TMA and TPO. TRA are sensitive markers of thyroid disease only at the extremes of thyroid function.

Keywords Thyroid disease - Population-based studies . Thyroid autoantibodies . Autoimmune thyroid disease . Autoantibody screening

\section{Introduction}

Circulating antibodies to thyroid antigens are easily measurable markers of thyroid autoimmunity, a leading cause of thyroid disease in the Western world. Thyroid microsomal antibodies (TMA), recently identified as antibodies against the cytoplasmic enzyme thyroid peroxidase (TPO) [1, 2], thyroglobulin antibodies (TGA) and thyroid-stimulating hormone (TSH) receptor antibodies (TRA) are widely used in the diagnosis of autoimmune thyroid diseases [3]. The prevalence of thyroid autoantibodies among patients with thyroid dysfunction varies according to the underlying case-mix of the patients thyroid disease $[4,5]$. For this reason the diagnostic sensitivity of thyroid autoantibodies should be assessed over the entire spectrum of thyroid dysfunction, including in those with undiagnosed disease, who are the 
majority of those with thyroid dysfunction in the general population [6]. Therefore, a population-based study provides a unique opportunity for estimating the diagnostic sensitivity of thyroid autoantibodies.

The aim of this study was to estimate the sensitivity of thyroid autoantibodies for the diagnosis of thyroid disease using data from the Cremona Study, a populationbased cross-sectional survey of the adult population of Northern Italy.

\section{Materials and methods}

\section{The Cremona Study}

The Cremona Study is a population-based, cross-sectional survey conducted in 1990 in the general population of Northern Italy [7]. In brief, 3,097 individuals aged $\geq 40$ years were randomly selected from the population of three municipalities of the Cremona Health District (Cremona, Casalbuttano and Vescovato). Of these, 2,096 individuals $(67 \%)$ agreed to participate in the survey. Thyroid dysfunction was assessed by measuring serum TSH in 2,019 participants for whom an unthawed serum aliquot was available. Thyroid autoantibodies (TMA, TPO, TGA and TRA), free triiodothyronine (fT3) and free thyroxine (fT4) were then measured in individuals in the bottom and top four percentiles of the TSH distribution calculated after exclusion of participants with treated thyroid disease (eight treated with thiamazole and two treated with levothyroxine).

Hormone and autoantibody measurements

All laboratory measurements were performed on samples frozen and maintained at $-20^{\circ} \mathrm{C}$ until assay. TSH, fT3 and fT4 were measured using an automated third-generation electrochemiluminescence method (Elecsys 2010 immunoassay system; Roche Diagnostics, Mannheim, Germany). TMA were measured by indirect haemoagglutination using a commercial kit (Serodia, Tokyo, Japan); the threshold for positivity was agglutination at a dilution $\geq 1: 100$. TPO were measured by radioimmunoassay using a commercial kit (Medipan, Berlin, Germany); the threshold for positivity was $60 \mathrm{U} / \mathrm{ml}$. TGA were measured by indirect haemoagglutination using a commercial kit (Serodia); the threshold for positivity was agglutination at a dilution $\geq 1: 100$. TRA were measured by radioimmunoassay using a commercial kit (TRAK human, Brahms, Berlin, Germany); the threshold for positivity was $1 \mathrm{U} / \mathrm{ml}$.

\section{Statistical analysis}

Prevalence rates were expressed as percentages with $95 \%$ confidence intervals $(95 \% \mathrm{CI})$. Sensitivity was the proportion of individuals with thyroid dysfunction who tested positive for a certain thyroid autoantibody. Agreement between thyroid antibodies was assessed using the percent agreement and $\mathrm{K}$ statistics [8]. The $\mathrm{K}$ statistic was interpreted as described by Byrt [9]. Data were analysed using Stata, version 9.0 (Stata Corporation, College Station, TX).

\section{Results}

TSH interval, median fT3 and fT4 for the top and bottom four percentiles of the TSH distribution are shown in Table 1. As expected, the majority of participants in the bottom and top four percentiles of the $\mathrm{TSH}$ distribution were women $(66.7 \%$ and $70.0 \%$, respectively).

The prevalence rates of thyroid antibodies in the four bottom and top percentiles of the TSH distribution are shown in Table 2. In the bottom four percentiles of the TSH distribution, the highest prevalence of thyroid anti-

Table 1 Selected percentiles of the TSH distribution in the Cremona study participants

\begin{tabular}{|c|c|c|c|c|c|c|c|}
\hline \multirow[t]{2}{*}{ TSH percentile } & \multirow[t]{2}{*}{ TSH interval (mIU/l) } & \multicolumn{2}{|c|}{ fT3 $(\mathrm{pmol} / \mathrm{l})^{\mathrm{a}}$} & \multicolumn{2}{|c|}{ fT4 $(\mathrm{pmol} / \mathrm{l})^{\mathrm{b}}$} & \multicolumn{2}{|c|}{ Sex } \\
\hline & & Median & Interquartile range & Median & Interquartile range & Female $(n=110)$ & Male $(n=51)$ \\
\hline $1 \mathrm{st}$ & $\leq 0.009$ & 8.22 & $6.95-9.87$ & 19.56 & $15.44-21.62$ & 17 & 5 \\
\hline 2nd & $0.009-0.180$ & 6.53 & $5.82-7.93$ & 17.89 & $16.73-19.82$ & 14 & 5 \\
\hline $3 \mathrm{rd}$ & $0.180-0.287$ & 5.45 & $4.82-6.14$ & 17.50 & $15.19-18.66$ & 12 & 8 \\
\hline 4th & $0.287-0.380$ & 5.01 & $4.65-5.67$ & 19.05 & $16.34-22.01$ & 11 & 9 \\
\hline 97th & $4.32-4.85$ & 5.70 & $5.01-6.31$ & 15.96 & $15.32-17.76$ & 8 & 12 \\
\hline 98th & $4.85-5.97$ & 5.51 & $5.14-5.90$ & 14.80 & $13.51-16.22$ & 17 & 3 \\
\hline 99th & $5.97-8.83$ & 5.48 & $5.04-6.73$ & 11.84 & $8.75-14.54$ & 17 & 3 \\
\hline 100th & $8.83-100.00$ & 5.10 & $4.68-5.51$ & 15.19 & $13.26-16.34$ & 14 & 6 \\
\hline
\end{tabular}

aNormal range: $3.08-6.16 \mathrm{pmol} / 1$

bNormal range: $9.01-21.88 \mathrm{pmol} / \mathrm{l}$ 
Table 2 Prevalence (percentages with 95\% confidence intervals in parentheses) of thyroid autoantibodies in the lower (1st, 2nd, 3rd and 4th) and upper (97th, 98th, 99th and 100th) percentiles of the TSH distribution among Cremona Study participants

\begin{tabular}{|c|c|c|c|c|c|c|c|c|}
\hline \multirow[t]{2}{*}{ Antibody } & \multicolumn{8}{|c|}{ Percentiles of the TSH distribution } \\
\hline & $1 \mathrm{st}$ & 2nd & $3 \mathrm{rd}$ & 4 th & 97 th & 98th & 99th & $100^{\text {th }}$ \\
\hline \multirow[t]{2}{*}{$\mathrm{TPO}^{\mathrm{a}}$} & 31.8 & 0.0 & 10.0 & 30.0 & 25.0 & 60.0 & 60.0 & 70.0 \\
\hline & $(13.9-54.9)$ & & $(1.2-31.7)$ & $(11.9-54.3)$ & $(8.7-49.1)$ & (36.1-80.9) & (36.1-80.9) & $(45.7-88.1)$ \\
\hline \multirow{2}{*}{$\mathrm{TMA}^{\mathrm{b}}$} & 31.8 & 10.5 & 5.0 & 30.0 & 25.0 & 45.0 & 60.0 & 75.0 \\
\hline & $(13.9-54.9)$ & $(1.3-33.1)$ & $(0.1-24.9)$ & $(11.9-54.3)$ & $(8.7-49.1)$ & $(23.1-68.5)$ & $(36.1-80.9)$ & $(50.9-91.3)$ \\
\hline \multirow[t]{2}{*}{$\mathrm{TGA}^{\mathrm{c}}$} & 27.3 & 5.3 & 5.0 & 5.0 & 20.0 & 20.0 & 50.0 & 50.0 \\
\hline & $(10.7-50.2)$ & $(0.1-26.0)$ & $(0.1-24.9)$ & $(0.1-24.9)$ & $(5.7-43.7)$ & $(5.7-43.7)$ & $(27.2-72.8)$ & $(27.2-72.8)$ \\
\hline \multirow[t]{2}{*}{$\mathrm{TRA}^{\mathrm{d}}$} & 31.8 & 5.3 & 0.0 & 0.0 & 5.0 & 10.0 & 0.0 & 25.0 \\
\hline & $(13.9-54.9)$ & $(0.1-26.0)$ & & & $(0.1-24.9)$ & $(1.2-31.7)$ & & $(8.6-49.1)$ \\
\hline \multirow[t]{2}{*}{ TPO and/or TMA } & 31.8 & 10.5 & 10.0 & 30.0 & 25.0 & 60.0 & 65.0 & 75.0 \\
\hline & $(13.9-54.9)$ & $(1.3-33.1)$ & $(1.2-31.7)$ & $(11.9-54.3)$ & $(8.7-49.1)$ & $(36.1-80.9)$ & $(40.8-84.6)$ & $(50.9-91.3)$ \\
\hline \multirow[t]{2}{*}{ Any } & 45.5 & 15.8 & 10.0 & 30.0 & 30.0 & 65.0 & 70.0 & 85.0 \\
\hline & $(24.4-67.8)$ & $(3.4-39.6)$ & $(1.2-31.7)$ & $(11.9-54.3)$ & $(11.9-54.3)$ & $(40.8-84.6)$ & $(45.7-88.1)$ & $(62.1-96.8)$ \\
\hline
\end{tabular}

aPositive if $\geq 60 \mathrm{U} / \mathrm{ml}$

bPositive at a dilution $\geq 1: 100$

'Positive at a dilution $\geq 1: 100$

dPositive if $\geq 1 \mathrm{U} / \mathrm{ml}$

bodies was observed in the first TSH percentile (TSH $\leq 0.009 \mathrm{mIU} / \mathrm{l})$. TMA, TPO and TRA had the highest prevalence $(31.8 \%, 95 \%$ CI $13.9-54.9 \%)$ and TGA the lowest $(27.3 \%, 95 \%$ CI $10.7-50.2 \%)$ and $45.5 \%$ of the participants were positive for at least one thyroid autoantibody. In the 2nd, 3rd and 4th percentile, TMA and TPO had the highest prevalence. In the upper four percentiles of the TSH distribution, the prevalence of any positive thyroid autoantibody increased from $30.0 \%$ (95\% CI $11.9-54.3 \%$ ) in the 97th percentile (TSH 4.32-4.85 $\mathrm{mIU} / \mathrm{L}$ ) to $85.0 \%$ (95\% CI $62.1-96.8 \%)$ ] in the 100th percentile (TSH 8.83-100.00 mIU/L). TMA and TPO had the highest prevalence, followed by TGA and TRA. In the top four percentiles of the TSH distribution, the highest prevalence of TRA was observed in the 100th percentile.

The values for the sensitivity of thyroid autoantibodies in the bottom and top percentiles of the TSH distribution are shown in Table 3. In the four bottom percentiles of the TSH distribution the sensitivity of thyroid autoantibodies was highest for TMA, followed by TPO, TGA and TRA. In the four top percentiles of the TSH distribution the sensitivity of thyroid autoantibodies was highest for TPO, followed by TMA, TGA and TRA.

TMA and TPO showed the best agreement between thyroid autoantibodies (percent agreement $94.4 \%$; kappa statistics $87.8 \%, 95 \%$ CI $80.1-95.5 \%$ ). The agreement between TMA and TPO was similar at both ends of the TSH distribution. In the bottom four percentiles, TMA and TPO were discordant in three patients: two were positive for TMA and one for TPO (percent agreement 96.3\%; kappa statistics $88.0 \%, 95 \%$ CI $74.8-100.0 \%$ ). In the top four percentiles, TMA and TPO were discordant
Table 3 Sensitivity of thyroid autoantibodies in the top and bottom percentiles of the TSH distribution among Cremona Study participants

\begin{tabular}{lrr}
\hline Antibody & \multicolumn{2}{c}{ Sensitivity (\%) } \\
\cline { 2 - 3 } & 1st to 4th percentile & 97th to 100th percentile \\
\hline TPO $^{\mathrm{a}}$ & 18.5 & 53.8 \\
TMA $^{\mathrm{b}}$ & 19.8 & 51.2 \\
TGA $^{\mathrm{c}}$ & 11.1 & 35.0 \\
TRA $^{\mathrm{d}}$ & 9.9 & 10.0 \\
TPO and/or TMA $_{\text {Any }}$ & 21.0 & 56.3 \\
\hline
\end{tabular}

aPositive if $\geq 60 \mathrm{U} / \mathrm{ml}$

bPositive at a dilution $\geq 1: 100$

'Positive at a dilution $\geq 1: 100$

dPositive if $\geq 1 \mathrm{U} / \mathrm{ml}$

in six patients: two were positive for TMA and four for TPO (percent agreement 92.5\%; kappa statistics $85.0 \%$, 95\% CI 73.4-96.5\%). In the bottom four percentiles of the TSH distribution, four patients negative for either TMA or TPO had other autoantibodies: two were positive for TRA, one for TGA and one for both TGA and TRA. In the bottom four percentiles of the TSH distribution, five patients negative for either TMA or TPO had other autoantibodies: three were positive for TGA and two for TRA.

\section{Discussion}

The aim of this study was to assess the diagnostic sensitivity of thyroid autoantibodies using data from a population-based study in adults. We measured four well-established thyroid autoantibodies in patients at the two 
extremes of thyroid function, including all those with thyroid dysfunction among participants in the Cremona Study, a population-based cross-sectional survey of the adult population of Northern Italy. The proportions of individuals who were positive to at least one autoantibody were $25.9 \%$ in the four TSH percentiles at the hyperthyroid end $(45.5 \%$ in the first percentile) and $62.5 \%$ in the four TSH percentiles at the hypothyroid end ( $85 \%$ in the 100th TSH percentile).

Although we did not measure urine iodine in our subjects, Italy is classified as a mildly iodine-deficient area, and in the largest proportion of those with thyroid dysfunction the condition is probably associated with thyroid autoimmunity [10]. Indeed the prevalence of thyroid autoantibodies among the Cremona Study participants was similar to that reported in the United Kingdom [11], United States [6], Australia [12], Norway [13], The Netherlands [14] and Denmark [15], all geographic regions with no or mild iodine deficiency.

The most frequently detected thyroid autoantibodies were TMA and TPO, which were present in the majority of individuals with hypothyroidism and in a significant proportion of individuals with hyperthyroidism. As expected, since TMA and TPO recognize the same autoantigen [1, 2], these two autoantibodies were in good agreement. TMA were slightly more sensitive than TPO at the hyperthyroid end, while the opposite was observed at the hypothyroid end. TGA were the next most sensitive antibodies at both extremes of the TSH distribution, while TRA were the least detected thyroid autoantibodies. However, the sensitivity of TRA was high and similar to that of TMA and TPO in the first percentile of the TSH distribution, which comprises individuals with clinically overt hyperthyroidism in which TRA identifies the subgroup affected by Graves' disease. The prevalence of TRA was also increased at the hypothyroid end of the TSH distribution $(25.0 \%$ in the 100 th percentile), confirming the dual stimulating/inhibiting action of this thyroid autoantibody and supporting its role in some cases of severe hypothyroidism.

In conclusion, among a representative sample of individuals with dysthyroidism, TMA and TPO were the most sensitive markers of thyroid disease, therefore being ideal for screening purposes. TGA only marginally increased the diagnostic sensitivity of TMA and TPO. TRA were not useful for the screening of autoimmune thyroid disease, but they have high sensitivity and clinical usefulness at the extremes of thyroid function.

Conflict of interest statement The authors declare that they have no conflict of interest related to the publication of this article.

\section{References}

1. Czarnocka B, Ruf J, Ferrand M et al (1985) Purification of the human thyroid peroxidase and its identification as the microsomal antigen involved in autoimmune thyroid diseases. FEBS Lett 190:147-152

2. Portmann L, Hamada N, Heinrich G, DeGroot LJ (1985) Antithyroid peroxidase antibody in patients with autoimmune thyroid disease: possible identity with anti-microsomal antibody. J Clin Endocrinol Metab 61:1001-1003

3. Sinclair D (2006) Clinical and laboratory aspects of thyroid autoantibodies. Ann Clin Biochem 43:173-183

4. Lind P, Langsteger W, Molnar M et al (1998) Epidemiology of thyroid diseases in iodine sufficiency. Thyroid 8:1179-1183

5. Laurberg P, Pedersen KM, Hreidarsson A et al (1998) Iodine intake and the pattern of thyroid disorders: a comparative epidemiological study of thyroid abnormalities in the elderly in Iceland and in Jutland, Denmark. J Clin Endocrinol Metab 83:765-769

6. Hollowell JG, Staehling NW, Flanders WD et al (2002) Serum TSH, T(4), and thyroid antibodies in the United States population (1988 to 1994): National Health and Nutrition Examination Survey (NHANES III). J Clin Endocrinol Metab 87:489-499

7. Garancini MP, Calori G, Ruotolo G et al (1995) Prevalence of NIDDM and impaired glucose tolerance in Italy: an OGTT-based population study. Diabetologia 38:306-313

8. Landis JR, Koch GG (1977) An application of hierarchical kappa-type statistics in the assessment of majority agreement among multiple observers. Biometrics 33:363-374

9. Byrt T (1996) How good is that agreement? Epidemiology 7:561

10. Vitti P, Delange F, Pinchera A et al (2003) Europe is iodine deficient. Lancet 361:1226

11. Vanderpump MP, Tunbridge WM, French JM et al (1995) The incidence of thyroid disorders in the community: a twenty-year follow-up of the Whickham Survey. Clin Endocrinol 43:55-68

12. O'Leary PC, Feddema PH, Michelangeli VP et al (2006) Investigations of thyroid hormones and antibodies based on a community health survey: the Busselton thyroid study. Clin Endocrinol 64:97-104

13. Bjoro T, Holmen J, Kruger O et al (2000) Prevalence of thyroid disease, thyroid dysfunction and thyroid peroxidase antibodies in a large, unselected population. The Health Study of NordTrondelag (HUNT). Eur J Endocrinol 143:639-647

14. Hoogendoorn EH, Hermus AR, de Vegt F et al (2006) Thyroid function and prevalence of anti-thyroperoxidase antibodies in a population with borderline sufficient iodine intake: influences of age and sex. Clin Chem 52:104-111

15. Bülow Pedersen I, Laurberg P, Knudsen N et al (2005) A population study of the association between thyroid autoantibodies in serum and abnormalities in thyroid function and structure. Clin Endocrinol 62:713-720 